\title{
Sustentabilidade agroindustrial: Processos Oxidativos Avançados (POA) utilizando bioadsorventes brasileiros
}

\author{
Agroindustrial sustainability: Advanced Oxidative Processes (AOP) using brazilian bioabsorbents
}

Sostenibilidad agroindustrial: Procesos Oxidativos Avanzados (POA) utilizando bioabsorbentes

brasileños

Recebido: 10/02/2021 | Revisado: 19/02/2021 | Aceito: 20/04/2021 | Publicado: 11/05/2021

\author{
Taynara Alvares Martins \\ ORCID: https://orcid.org/0000-0002-6559-3762 \\ Universidade Federal de Goiás, Brasil \\ E-mail: taynaramartinsa@gmail.com \\ Maria Carolina de Almeida \\ ORCID: https://orcid.org/0000-0002-6822-4866 \\ Universidade Federal de Goiás, Brasil \\ E-mail: maria.almeida@ifg.edu.br \\ Isabely Crysis Gonçalves Lopes \\ ORCID: https://orcid.org/0000-0002-7610-9382 \\ Universidade Federal de Goiás, Brasil \\ E-mail: isabelycgl@gmail.com \\ Isabela Ferreira Moreno \\ ORCID: https://orcid.org/0000-0002-4049-6165 \\ Universidade Federal de Goiás, Brasil \\ E-mail: isabelafmoreno@gmail.com \\ Tatianne Ferreira de Oliveira \\ ORCID: https://orcid.org/0000-0002-8852-5471 \\ Universidade Federal de Goiás, Brasil \\ E-mail: tatianne_ferreira_oliveira@ufg.br
}

\begin{abstract}
Resumo
O objetivo deste trabalho foi avaliar bioadsorventes originários de resíduos agroindustriais brasileiros (casca de banana e mandioca) a serem utilizados na eliminação do poluente modelo azul de metileno (MB). Foram estudados neste contexto o processo de adsorção em diversas condições experimentais (temperatura de 25 a $50^{\circ} \mathrm{C}$, massa do bioadsorventes variando de 0,5 a $1,0 \mathrm{~g}$ ), assim como a modelagem cinética utilizando três diferentes modelos: pseudoprimeira ordem, pseudosegunda ordem e difusão intrapartícula. Foi estudado posteriormente os Processos Oxidativos Avançados (POA) UVc-AC e UV-C/Casca em diferentes condições (temperatura de 25 a $50^{\circ} \mathrm{C}$, e potência da lâmpada 11 a $33 \mathrm{~W}$ ). Os resultados obtidos foram promissores, onde os melhores resultados nos processos de adsorção foram aqueles utilizando o bioadsorvente casca de banana, com uma massa de $0,5 \mathrm{~g}$ e uma temperatura de $50{ }^{\circ} \mathrm{C}$. Para os POAs utilizados, a melhor cinética de degradação do $\mathrm{MB}$ foi na condição $\mathrm{C} 4\left(55^{\circ} \mathrm{C}, 33 \mathrm{~W}\right)$ utilizando o resíduo casca de banana, mostrando um resíduo agroindustrial satisfatório e eficiente á ser aplicado em combinação com o UV, contribuindo para a sustentabilidade agroindustrial e para a melhoria dos parâmetros de qualidade da água. Palavras-chave: Resíduo brasileiro; Casca de banana; Casca de mandioca; POAs; Mecanismos de adsorção.
\end{abstract}

\begin{abstract}
The objective of this work was to evaluate bioadsorbers from Brazilian agro-industrial residues (banana and cassava peel) to be used in the elimination of the pollutant blue methylene model (MB). In this context, the adsorption process under various experimental conditions (temperature from 25 to $50^{\circ} \mathrm{C}$, mass of the bio-adsorbents ranging from 0.5 to $1.0 \mathrm{~g}$ ) was studied, as well as the kinetic modeling using three different models: pseudo-first order, pseudo-second intraparticle order and diffusion. Advanced Oxidative Processes (POA) UVc-AC and UV-C / Shells were subsequently studied under different conditions (temperature from 25 to $50^{\circ} \mathrm{C}$, and lamp power from 11 to $33 \mathrm{~W}$ ). The results obtained were promising, where the best results in the adsorption processes were those using the bioabsorbent banana peel, with a mass of $0.5 \mathrm{~g}$ and a temperature of $50^{\circ} \mathrm{C}$. For the POAs used, the best MB degradation kinetics was in the $\mathrm{C} 4$ condition $\left(55^{\circ} \mathrm{C}, 33 \mathrm{~W}\right)$ using the banana peel residue, showing a satisfactory and efficient agroindustrial residue to be applied in combination with the UV, contributing to the agro-industrial sustainability and for the improvement of water quality parameters.
\end{abstract}

Keywords: Brazilian waste; Banana peel; Cassava peel; AOPs; Adsorption mechanisms. 


\begin{abstract}
Resumen
El objetivo de este trabajo fue evaluar bioabsorbentes de residuos agroindustriales brasileños (cáscara de banano y yuca) para ser utilizados en la eliminación del contaminante modelo azul metileno (MB). En este contexto, se estudió el proceso de adsorción en diversas condiciones experimentales (temperatura de 25 a $50{ }^{\circ} \mathrm{C}$, masa de los bioadsorbentes de 0,5 a 1,0 g), así como el modelado cinético utilizando tres modelos diferentes: orden intrapartículas y difusión. Posteriormente se estudiaron los Procesos Oxidativos Avanzados (POA) UVc-AC y UV-C / Peel en diferentes condiciones (temperatura de 25 a $50^{\circ} \mathrm{C}$ y potencia de lámpara de 11 a $33 \mathrm{~W}$ ). Los resultados obtenidos fueron prometedores, donde los mejores resultados en los procesos de adsorción fueron los que utilizaron la cáscara de plátano bioabsorbente, con una masa de 0,5 gy una temperatura de $50^{\circ} \mathrm{C}$. Para los POA utilizados, la mejor cinética de degradación del $\mathrm{MB}$ fue en la condición $\mathrm{C} 4\left(55^{\circ} \mathrm{C}, 33 \mathrm{~W}\right)$ utilizando el residuo de cáscara de banano, mostrando un residuo agroindustrial satisfactorio y eficiente para ser aplicado en combinación con la UV, contribuyendo a la agroindustria. sostenibilidad industrial y para la mejora de los parámetros de calidad del agua.
\end{abstract}

Palabras clave: Residuos brasileños; Cáscara de plátano, Cáscara de yuca, POAs, Mecanismos de adsorción.

\title{
1. Introdução
}

O agronegócio passou a ser o principal componente da pauta das exportações e vem contribuindo para o equilíbrio da balança comercial brasileira. Com ele temos uma vasta quantidade e diversidade de resíduos originários da agroindústria e do agronegócio brasileiro. Os principais resíduos gerados no processamento de frutas e hortaliças, por exemplo, por exemplo, são a casca, caroço ou sementes e bagaço (Vieira filho e Fishlow, 2017).

O setor bananeiro ocupa lugar de destaque no cenário sócio-econômico brasileiro e contribui com uma grande porcentagem do valor da produção dos principais produtos agrícolas. É a fruta mais consumida no mundo, tanto pelas classes privilegiadas como pelas menos favorecidas (Aguiar, 2006). Por outro lado, a mandioca (Manihot suculenta Crantz) é raíz mais consumida em todo o Brasil, cultivada em todos os estados do país e apresenta grande importância na alimentação brasileira, gerando igualmente uma quantidade enorme de resíduos (Iheukwumere et al., 2008).

Os corantes químicos artificiais são compostos sintéticos com uma cor específica que pode ser quimicamente ligada ao substrato (por exemplo, fibra, papel, tecido) para fornecer uma cor bonita. Eles têm sido aplicados em vários campos do setor industrial (por exemplo, têxtil, papel, impressão, plásticos, couro) e na vida diária (por exemplo, alimentos, bebidas, medicamentos, cosméticos, produtos pessoais) (Pang et al., 2019; Li et al., 2019).As moléculas de corantes nas águas residuais levam à mutagenicidade, carcinogenicidade e disfunção do rim, fígado, cérebro, sistema reprodutor e sistema nervoso central dos seres humanos (Zhou et al., 2019).

Por isso, a remoção destes corantes antes do descarte do efluente industrial tem grande relevância do ponto de vista ambiental. O descarte responsável desses corantes é um grande desafio, processos de tratamento mais eficaz e economicamente eficiente precisam ser desenvolvidos. Essa tarefa é desafiadora, pois as concentrações dos corantes em águas residuais precisam ser muito baixas antes da liberação no meio ambiente (Islam et al., 2019).

Águas residuárias contendo corantes apresentam características de composições complexas e variáveis, grande descarga com ampla distribuição de moléculas de corantes dissolvidas que são difíceis de serem degradadas (Yagub et al., 2014). Se essas águas residuais forem descartadas sem tratamento, causarão sérios danos ao meio ambiente (SANTHOSH et al., 2016). Em particular, o azul de metileno (MB - methylene blue), composto aromático heterocíclico com uma fórmula molecular $\mathrm{C}_{16} \mathrm{H}_{18} \mathrm{~N}_{3} \mathrm{SCl}$ é um corante catiônico característico que tem sido amplamente utilizado em indústrias como o tingimento de algodão, acrílico, cânhamo, seda, papel e tintas de fabricação devido à sua excelente solubilidade em água e estabilidade de cor (Fadilhhad et al., 2019; Zhang et al., 2011). Dados da Organização Mundial da Saúde (OMS) classificaram o azul de metileno como contaminantes prioritários (Ezzatahmadi et al., 2017).

O processo de adsorção tem sido amplamente aplicado para descoloração da água em comparação com outras técnicas de tratamento físico-químico (De Souza et al., 2018). Estudos com a substituição dos convencionais carvões ativados por 
adsorventes naturais ou bioadsorventes a partir de resíduos da agroindústria como sementes de mamão, grãos de café, casca de coco, cascas de banana, batata e pepino, estão sendo aplicados para a remoção de poluentes da água (Shooto et al., 2019).

Em paralelo também nota-se nas últimas décadas, esforços de pesquisa no desenvolvimento de tecnologias mais eficazes para remover poluentes orgânicos persistentes provenientes de águas residuais. Entre estes processos destacam-se os Processos Oxidativos Avançados (POA's), capazes de gerar radicais hidroxila em quantidades suficientes para oxidar as substâncias químicas presentes em efluentes. Os POA's são oferecidos como uma alternativa aos métodos de oxidação convencionais e são baseados na geração de radicais hidroxila (• OH) (Flouret et al., 2018) e demonstram ser métodos de tratamento eficazes para degradar materiais persistentes quando os tratamentos convencionais não atingem a eficácia necessária. Estes processos podem ser heterogêneos ou homogêneos, e utilizar ou não a radiação UV (Habib et al., 2020).

Neste contexto, o objetivo deste trabalho foi elaborar bioadsorventes a partir de resíduos agroindustriais brasileiros (casca de banana e de mandioca); investigar a eficiência destes materiais no processo de adsorção e em Processos Oxidativos Avançados (POA) para a eliminação do poluente modelo corante azul de metileno.

\section{Metodologia}

\subsection{Material e caracterização do carvão ativado}

O carvão ativado (CA) utilizado neste estudo foi o comercial fornecido pela FBC Fábrica de Catalisadores, Brasil e resíduos de cascas de banana e mandioca provenientes do restaurante universitário da Universidade Federal de Goiás (UFG). O azul de metileno (MB) da marca Aldrich Chemistry apresenta pureza de 99,5\%. Os espectros de adsorção na região do infravermelho foram obtidos em espectrofotômetro (Perkinelmer, modelo Spectrum 400). As análises concentraram-se na região do infravermelho entre 4000 e $400 \mathrm{~cm}^{-1}$ com resolução de $4 \mathrm{~cm}^{-1}$. As análises em MEV foram realizadas em Microscópio Eletrônico de Varredura Eletrônica (Jeol, modelo JSM - 6610) com o objetivo de avaliar o carvão ativado.

\subsection{Experimentos de Adsorção}

As cinéticas de adsorção foram investigadas para verificar a eficiência dos resíduos de cascas de banana e mandioca. Estas foram secas em estufa (Tecnal TE-394/3, Piracicaba-SP, Brasil) a temperatura de $40{ }^{\circ} \mathrm{C}$ até peso constante antes dos experimentos de adsorção.

As condições experimentais foram aplicadas em um reator erlenmeyer com $250 \mathrm{~mL}$ do efluente, na concentração do micropoluente de $0,2 \mathrm{~g} \mathrm{~L}^{-1}$ escolhida como padrão. O reator foi colocado em equipamento agitador Tecnal modelo TE424 com controle de agitação regulado para $150 \mathrm{rpm}$ conforme adaptação da metodologia proposta por de Moraes (2017). As amostras foram coletadas em diferentes intervalos de tempo $(0,5,10,15,25,35,45,60,80,100,120$ minutos) com o objetivo de avaliar a eliminação do poluente na solução. Após cada coleta, as amostras foram filtradas com papel filtro qualitativo e submetidas à análise em espectrofotômetro UV-visível (Femto, 700 plus) a $\lambda=265 \mathrm{~nm}$ (comprimento de onda do MB).

Foi realizado um planejamento experimental com 2 variáveis independentes: massa do CA e temperatura (Tabela 1), com o objetivo de escolher o melhor resíduo a ser utilizado no Processo Oxidativo Avançado. As análises foram feitas em duplicata. A capacidade de adsorção $\left(\mathrm{mg} \mathrm{g}^{-1}\right)$ foi calculada pela Equação 1.

$$
q_{t}=\frac{\left(C_{o}-C_{t}\right) \cdot V}{m} 10^{-3}(1)
$$

em que $\mathrm{q}_{\mathrm{t}}=$ capacidade de adsorção de $\mathrm{CA}\left(\mathrm{mg} \mathrm{g}^{-1}\right) ; \mathrm{C}_{\mathrm{o}}$ e $\mathrm{C}_{\mathrm{t}}=$ concentrações do poluente $\left(\mathrm{g} \mathrm{L}^{-1}\right)$, respectivamente no estado inicial e no instante $\mathrm{t}(\mathrm{min}) ; \mathrm{V}=$ volume da solução $(\mathrm{L}) ; \mathrm{m}=$ a massa do carvão ativado (g). 
Tabela 1. Processo de adsorção: fatores de influência investigados.

\begin{tabular}{llll}
\hline Fatores & Código & $\mathbf{- 1}$ & $\mathbf{1}$ \\
\hline Massa CA $(\mathrm{g})$ & $\mathrm{x}_{1}$ & 0,5 & 1,0 \\
Temperatura $\left({ }^{\circ} \mathrm{C}\right)$ & $\mathrm{x}_{2}$ & 25 & 50 \\
\hline
\end{tabular}

Fonte: Autores.

\subsection{Modelos matemáticos}

A modelagem cinética foi realizada utilizando o modelo de pseudo-primeira ordem (Stoeckli et al., 2001), representado pela Equação 2, pseudo-segunda ordem (Boehm, 2002) Equação 3 e difusão intrapartícula (Rivera-Utrilla e Sánchez-polo, 2002) Equação 4.

$\frac{d q_{t}}{d t}=k_{1}\left(q_{e}-q_{t}\right)$

$\frac{\mathrm{dq}}{\mathrm{dt}}=\mathrm{k}_{2}\left(\mathrm{q}_{\mathrm{e}}-\mathrm{q}_{\mathrm{t}}\right)^{2}$

$\mathrm{q}_{\mathrm{t}}=\mathrm{k}_{3} \mathrm{t}^{0{ }_{\mathrm{g}} 5}+\mathrm{c}$

Em que $\mathrm{q}_{\mathrm{e}}$ é a quantidade de soluto adsorvido por unidade de massa de adsorvente (mg.g $\left.\mathrm{g}^{-1}\right)$; $\mathrm{q}_{\mathrm{t}}$ é a quantidade de soluto adsorvido por unidade de massa de adsorvente $\left(\mathrm{mg}_{\mathrm{g}} \mathrm{g}^{-1}\right)$ no tempo $\mathrm{t}(\mathrm{min}) ; \mathrm{k}_{1}$ é a constante cinética de pseudo-primeira ordem $\left(\mathrm{min}^{-1}\right) ; \mathrm{k}_{2}$ é a constante cinética de pseudo-segunda ordem $\left(\mathrm{g} \cdot \mathrm{mg}^{-1} \cdot \mathrm{min}^{-1}\right)$; $\mathrm{k}_{3}$ é a constante cinética de difusão intrapartícula $\left(\mathrm{mg} \cdot \mathrm{g}^{-1} \cdot \mathrm{min}^{-0,5}\right)$ e c é a constante relacionada à espessura da camada de difusão interna ou externa (mg.g $\left.{ }^{-1}\right)$.

\subsection{Acoplamento UV-C /CA e acoplamento UV-C/CASCAS}

Foi realizado um planejamento fatorial completo (Tabela 2), com 3 repetições no ponto central, totalizando 7 testes em cada, variando a potência da lâmpada $(11,22$ e $33 \mathrm{~W})$ e a temperatura $\left(15,35\right.$ e $\left.55^{\circ} \mathrm{C}\right)$ com a massa de $0,5 \mathrm{~g}$ de carvão ativado (massa escolhida nos experimentos de adsorção). $150 \mathrm{~mL}$ de solução contendo $0,2 \mathrm{gL}^{-1}$ de $\mathrm{MB}$ e $0,5 \mathrm{~g}$ de $\mathrm{CA}$ foram colocados em cada erlenmeyer sob agitação constante a 200 rpm em um agitador magnético (FISATOM, modelo 752), por 2 horas e submetidos à radiação ultravioleta (UV-C ), radiação emitida por lâmpadas localizadas a $10 \mathrm{~cm}$ da superfície da solução. Alíquotas de $10 \mathrm{~mL}$ foram coletadas nos seguintes tempos: 0, 6, 12, 24, 36, 48, 60, 80, 100 e 120 minutos. Após cada coleta, $5 \mathrm{~mL}$ de sulfito de sódio $\left(0,1\right.$ mol.L $\left.\mathrm{L}^{-1} \mathrm{Na}_{2} \mathrm{SO}_{3}\right)$ foram adicionados para cessar a reação de oxidação e analisados por cromatógrafo (SHIMADZU, modelo LC 8A) a 664 nm (de Almeida, 2020). A melhor condição obtida nos processos UVC/CA foi aplicada com a cascas de banana e mandioca para análise da performance destes resíduos agroindustriais.

Coeficientes de regressão e valores de p foram determinados usando o programa Statistica 7.0 e foram determinados quais fatores e interações foram significativos a $5 \%$.

Tabela 2. UV-C/CA processo de acoplamento: fatores de influência investigados.

\begin{tabular}{lcccc}
\hline Fatores & código & $\mathbf{- 1}$ & $\mathbf{0}$ & $\mathbf{1}$ \\
\hline Potência da lampâda $(\mathrm{W})$ & $\mathrm{x}_{1}$ & 11 & 22 & 33 \\
Temperatura $\left({ }^{\circ} \mathrm{C}\right)$ & $\mathrm{x}_{2}$ & 15 & 35 & 55 \\
\hline
\end{tabular}

Fonte: Autores. 


\subsection{Análises de adsorção}

As amostras dos processos de adsorção foram analisadas em espectrofotômetro (FEMTO, modelo 700 plus) na região do ultravioleta, com $\lambda=664 \mathrm{~nm}$. Um cromatógrafo (SHIMADZU, modelo LC 8A) foi usado para determinar MB nos processos de UV-C /CA e UV-C/UV/carvão ativado/ tert-butanol usando um detector de arranjo de diodo (SHIMADZU, modelo SPD 20A), com o sistema em $664 \mathrm{~nm}$ no modo isocrático e fase reversa C-18 (SGE, WAKOSIL) (5 $\mu \mathrm{m}, 4,6 \mathrm{~mm}$ x 250 $\mathrm{mm}$ ). A fase móvel consistiu de uma solução aquosa de acetonitrila 70\% e 30\% de água ultra pura, com vazão de 1 mL.min-1 e injeção manual de $25 \mu \mathrm{L}$ de amostra.

\section{Resultados e Discussão}

\subsection{Processo de adsorção usando as cascas}

Para determinação da eficiência dos adsorventes na remoção da cor no processo de adsorção da solução do corante azul de metileno, foram realizadas medidas de absorbância dos experimentos nas diferentes condições (concentração do adsorvente e temperatura). Os resultados das cinéticas de adsorção dos bioadsorventes casca de banana e de mandioca na eliminação do azul de metileno (MB) são apresentadas na Figura 1 e Tabela 3.

Figura 1. Capacidades de adsorção (mg g-1) em função do tempo (minutos) das cinéticas de adsorção do azul de metileno (MB).

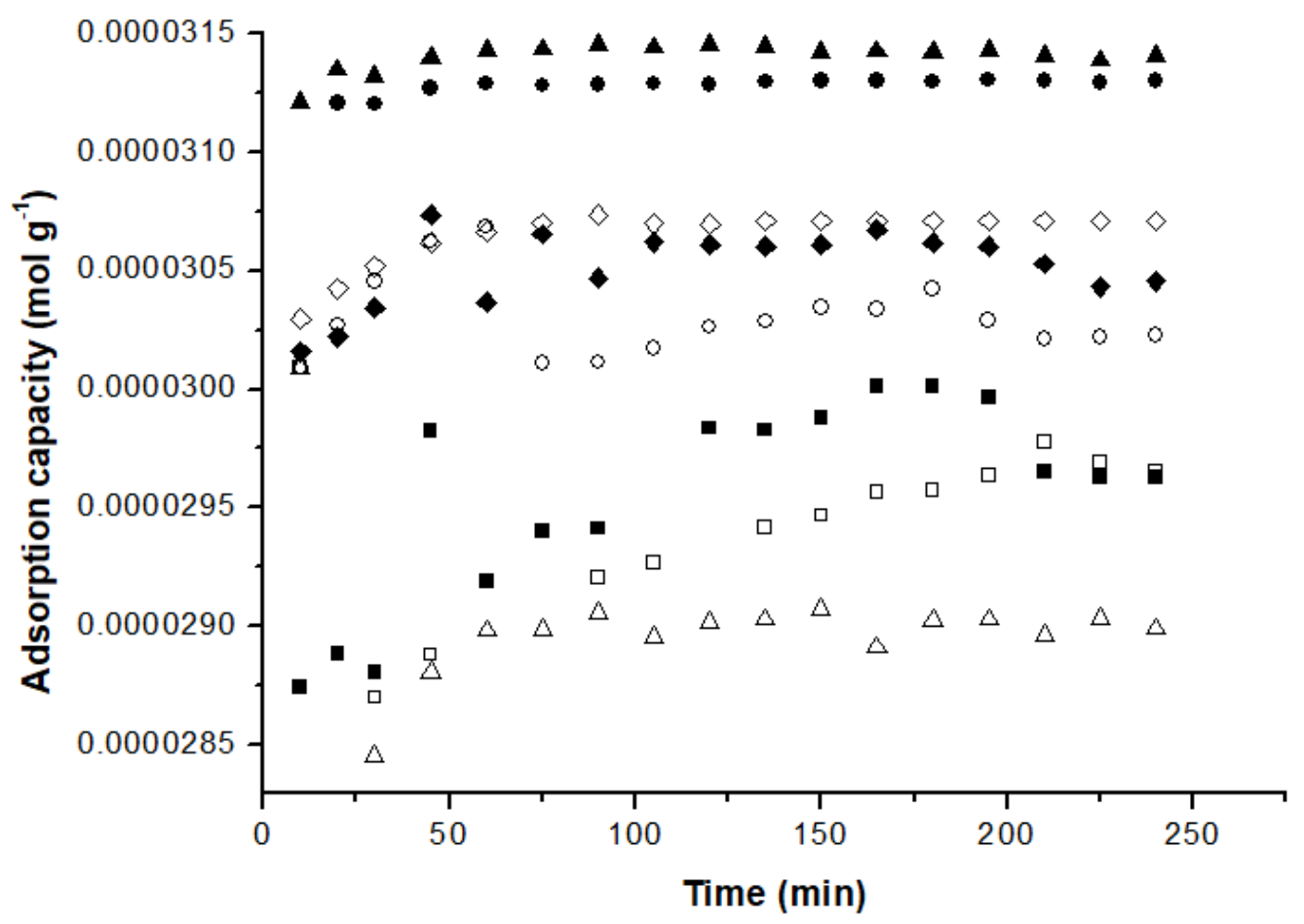

Legenda: Casca de banana: B1 (•) $0,5 \mathrm{~g}, 25^{\circ} \mathrm{C}$; B2 (•) $0,5 \mathrm{~g}, 50^{\circ} \mathrm{C} \mathrm{B} 3(\boldsymbol{\Delta}) 1,0 \mathrm{~g}, 25^{\circ} \mathrm{C}, \mathrm{B} 4(\bullet) 1,0 \mathrm{~g}, 50^{\circ} \mathrm{C}$.

Casca de mandioca: M1 () $0,5 \mathrm{~g}, 25^{\circ} \mathrm{C} \mathrm{M} 2(\circ) 0,5 \mathrm{~g}, 50^{\circ} \mathrm{C} \mathrm{M} 3(\Delta) 1,0 \mathrm{~g}, 25^{\circ} \mathrm{C}, \mathrm{M} 4(\diamond) 1,0 \mathrm{~g}, 50^{\circ} \mathrm{C}$.

Fonte: Autores. 
Tabela 3. Respostas obtidas no delineamento experimental de adsorção.

\begin{tabular}{ccccc}
\hline Ensaio & Massa de CA $(\mathbf{g})$ & ${\text { Temperatura }\left({ }^{\circ} \mathbf{C}\right)}^{\text {Qe }\left(\mathbf{m o l ~ g -}^{-1}\right)}$ & $\begin{array}{c}\text { Qe }\left(\mathbf{m o l ~ g}^{-1}\right) \\
\text { Casca de banana }\end{array}$ & $\begin{array}{c}{ }^{-1} \\
\text { Casca de mandioca }\end{array}$ \\
\hline 1 & 0,5 & 25 & $2,96 \mathrm{E}-05$ & $2,96 \mathrm{E}-05$ \\
2 & 0,5 & 50 & $3,13 \mathrm{E}-05$ & $3,02 \mathrm{E}-05$ \\
3 & 1,0 & 25 & $3,14 \mathrm{E}-05$ & $2,89 \mathrm{E}-05$ \\
4 & 1,0 & 50 & $3,04 \mathrm{E}-05$ & $3,07 \mathrm{E}-05$ \\
\hline
\end{tabular}

Fonte: Autores.

De acordo com os resultados obtidos a melhor condição experimental foi o experimento 2, utilizando uma massa de CA de $0,5 \mathrm{~g}$ e uma temperatura de $50{ }^{\circ} \mathrm{C}$. Observou-se também que as maiores capacidade de adsorção foram obtidas quando utilizou-se o bioadsorvente casca de banana, sendo um material que favorece este processo neste estudo.

Alver et al. (2020) estudaram a adsorção de azul de metileno em bio-composto de alginato magnético (casca de arroz) e demonstraram que a capacidade de adsorção experimental máxima foi calculada de $274,9 \mathrm{mg} / \mathrm{g}$, e concluiram que os vários parâmetros do processo, como pH, temperatura e concentração inicial do azul de metileno, influenciam na remoção total do poluente.

Ponnusami, Vikram e Srivastava (2008), realizaram ensaios de adsorção de azul de metileno em pó de folha da goiaba e observaram que o aumento da massa de adsorvente (incremento de $0,5 \mathrm{~g}$ para $2 \mathrm{~g}$ ) aumentou o percentual de remoção do adsorbato de 62,1 \% para 97,89\%. Uddin et al. (2009), estudaram a potencialidade dos resíduos de chá para a remoção adsortiva do azul de metileno em solução aquosa, eles constataram que o percentual de remoção e a concentração de adsorbato na superfície do adsorvente sofreram influência da dose de adsorvente.

Parvin et al. (2019) utilizaram a casca de coco como adsorvente para a remoção do azul de metileno e concluíram que a dosagem de adsorventes, influência na remoção do corante, uma vez que neste estudo o aumento da dosagem de $0,5 \mathrm{~g}$ para 1,5 g foram responsáveis pelo aumento no nível de remoção de $\mathrm{MB}$, sendo que o nível mais alto ( 86\%) de remoção de foi alcançado usando adsorvente de a uma concentração de 1,5 g / $\mathrm{L}$.

Normalmente, o aumento da dose de adsorvente fornece uma área de superfície maior e aumenta a disponibilidade dos locais de ligação (Kousha et al., 2012), ou seja, incremento da dose de adsorvente aumenta a área de adsorção por conta do maior número de sítios disponíveis. Por esse motivo, o aumento da quantidade de adsorvente e a manutenção da concentração fixa de adsorbato disponibilizam um grande número de locais para uma concentração fixa de adsorbato, consequentemente, aumentou a porcentagem de adsorção. Além disso, o aumento da massa também faz com que alguns sítios presentes na superfície do adsorvente não sejam ocupados por não serem energeticamente favoráveis, diminuindo assim o valor de qe (Reddy et al., 2016).

A otimização da quantidade de adsorvente para a adsorção de corantes é um parâmetro essencial para regular a capacidade de absorção de um adsorvente nas condições de estudos. Zare et al. (2016) demonstraram que a eficiência da remoção aumenta enquanto a capacidade de adsorção diminui com a dose crescente de adsorvente se outras condições permanecerem constantes.

A temperatura é um parâmetro importante no processo de adsorção. De acordo com Somsesta et al. (2020) o incremento de temperatura pode acelerar a mobilidade das partículas de corante e melhorar a interação entre os adsorventes e os pontos de adsorção na superfície do adsorvente, fornecendo energia suficiente. Além disso, a temperaturas mais altas, um 
efeito de intumescimento da estrutura interna do adsorvente pode permitir a passagem de moléculas de corante, resultando em um aumento da adsorção do azul de metileno.

Os estudos de adsorção foram realizados em diferentes temperaturas 25 e $50^{\circ} \mathrm{C}$. A capacidade de adsorção aumentou com o aumento da temperatura, indicando que a adsorção é provavelmente um processo endotérmico. Isto pode ser um resultado do aumento da mobilidade do corante com o aumento da temperatura (Alkan e Dogan, 2003). Um número crescente de moléculas também podem adquirir energia suficiente para passar por uma interação com sítios ativos na superfície. Além disso, o aumento da temperatura pode produzir um efeito de inchaço dentro da estrutura interna do adsorvente permitindo grande molécula corante para penetrar ainda mais (Asfour, 1985).

Li et al. (2020) estudaram a adsorção de corante azul de metileno em um carvão ativado à base de casca de noz de soluções aquosas, e demonstraram que a temperatura promoveu a capacidade de adsorção e este parâmetro operacional teve um efeito notável. O aumento da capacidade de adsorção do corante em função da temperatura pode estar associada ao incremento da mobilidade de moléculas azul de metileno com temperatura, promovendo a interação do corante com os principais locais receptores de adsorção na superfície do adsorvente.

\subsection{Modelo cinético}

A avaliação dos modelos cinéticos foi feita pela análise dos coeficientes de determinação $\left(\mathrm{R}^{2}\right)$ e pela proximidade dos dados experimentais e previstos pelos modelos cinéticos. O modelo de pseudo-segunda ordem ajustou-se melhor para a maioria dos testes. De acordo com Belaid et al., (2013) o modelo de pseudo-segunda ordem indica que a quimissorção é possivelmente o mecanismo de reação predominante entre o adsorvente e o adsorbato, envolvendo troca de elétrons ou forças de valência. Os resultados desses autores, para adsorção de fosfato por carvão ativado, também se ajustaram melhor ao modelo de pseudo-segunda ordem. Assim, os resultados observados na modelagem cinética indicam que pode ocorrer uma quimissorção.

Tabela 4. Parâmetros cinéticos dos modelos utilizados e aplicado ao tratamento de adsorção de $\mathrm{MB}$ em $\mathrm{CA}, \mathrm{B}=$ casca de banana; M- casca de mandioca.

\begin{tabular}{|c|c|c|c|}
\hline$\overline{C A}$ & $\begin{array}{l}\text { Modelo pseudo- } \\
\text { primeira ordem } \\
\qquad \mathbf{R}^{2}\end{array}$ & $\begin{array}{c}\text { Modelo pseudo- } \\
\text { segunda ordem } \\
\mathbf{R}_{2}{ }^{2}\end{array}$ & $\begin{array}{c}\text { Modelo } \\
\text { intrapartícula } \\
\mathbf{R}_{\mathbf{3}}{ }^{2}\end{array}$ \\
\hline B1 & 0.937 & 0.999 & 0.869 \\
\hline B2 & 0.847 & 0.999 & 0.971 \\
\hline B3 & 0.831 & 0.998 & 0.870 \\
\hline B4 & 0.809 & 0.998 & 0.839 \\
\hline M1 & 0.748 & 0.999 & 0.988 \\
\hline M2 & 0.548 & 0.999 & 0.981 \\
\hline M3 & 0.950 & 0.996 & 0.866 \\
\hline M4 & 0.847 & 0.987 & 0.967 \\
\hline
\end{tabular}

Fonte: Autores. 


\subsection{Acoplamento UV-C /CA e acoplamento UV-C/CASCAS}

A Figura 2 apresenta as curvas cinéticas da acoplagem utilizando POA (UV-C/CA e UV-C/BA) na eliminação do azul de metileno (MB).

Figura 2. Curvas cinéticas obtidas plotando-se a Concentração azul de metileno (mol L ${ }^{-1}$ ) em função do tempo (minutos).

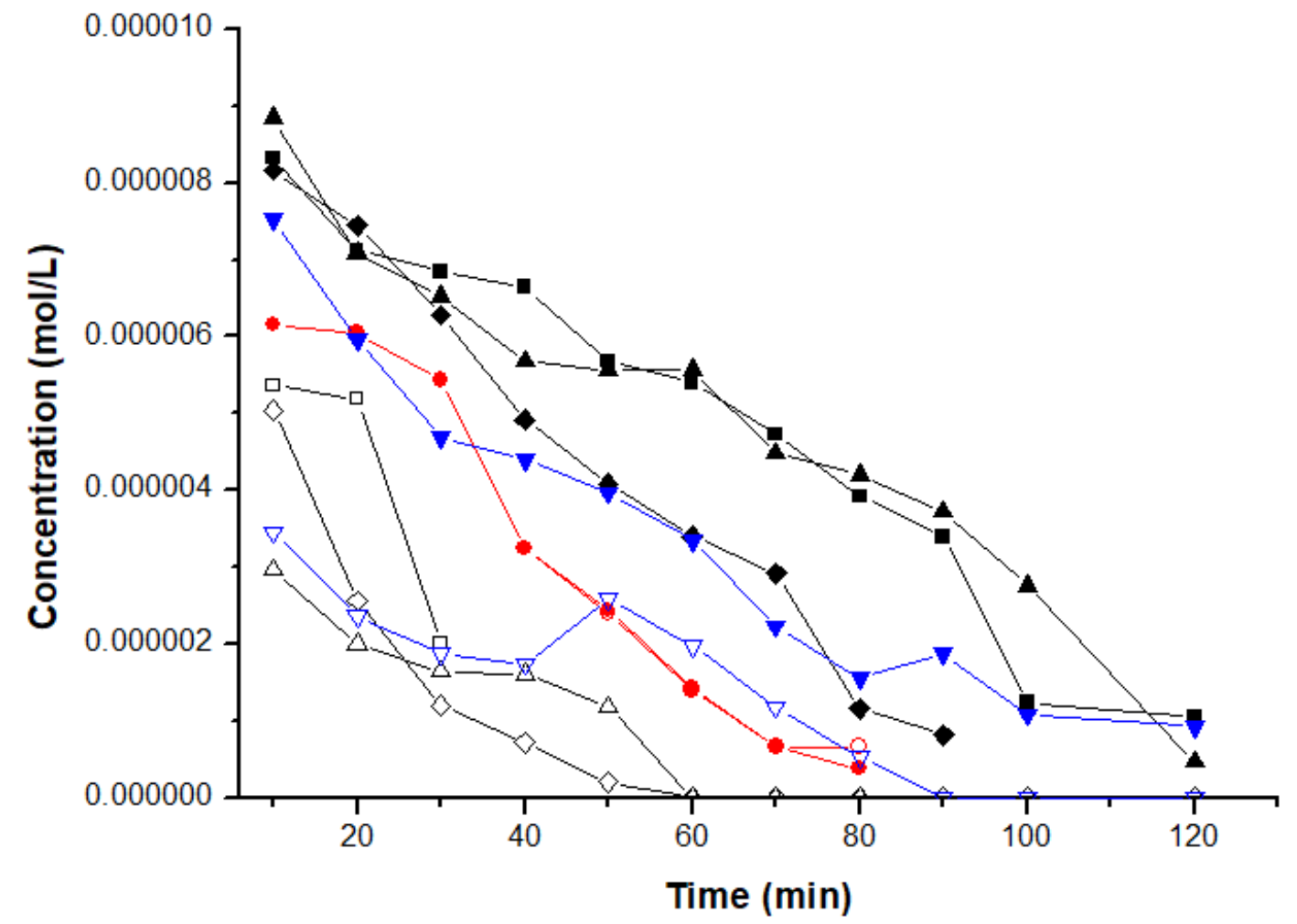

Legenda: $\mathrm{C} 1(\bullet \mathrm{CA} / \square \mathrm{Casca}): 15^{\circ} \mathrm{C}, 15 \mathrm{~W} ; \mathrm{C} 2(\bullet \mathrm{CA} \circ \mathrm{Casca}): 55^{\circ} \mathrm{C}, 15 \mathrm{~W} ; \mathrm{C} 3(\Delta \mathrm{CA} / \Delta \mathrm{Casca}): 15{ }^{\circ} \mathrm{C}, 33 \mathrm{~W} ; \mathrm{C} 4(\bullet \mathrm{CA} / \diamond \mathrm{Casca}) 55$ ${ }^{\circ} \mathrm{C}, 33 \mathrm{~W}$; C5 ( $\left.\nabla \mathrm{CA} / \mathrm{Casca}\right) 35^{\circ} \mathrm{C}, 22 \mathrm{~W}$; sendo $\mathrm{C} 5$ a média de $\mathrm{C} 5, \mathrm{C} 6$ e $\mathrm{C} 7$.

Fonte: Autores.

Os resultados obtidos na Figura 2 revelaram que a cinética de degradação do azul de metileno foi mais rápida quando utilizou-se POA acoplado com a casca de banana, mostrando um resíduo agroindustrial satisfatório e eficiente á ser aplicado em combinação com o UV. Processos oxidativos avançados quando acoplados com outros materiais apresentam melhores resultados quando separadamente, o que foi claramente mostrado na Figura 2. O ataque de radicais hidroxilas $\bullet \mathrm{OH}$ é um mecanismo muito importante envolvido na degradação de moléculas poluentes em fase aquosa. Alguns estudos revelam que um processo híbrido que combina elevada capacidade de oxidação e a elevada capacidade de adsorção do CA pode ser um processo bastante eficiente comparando com o POA convencional (Rivera-utrilla et al., 2006, Coha et al., 2021)

Observa-se que houve uma eliminação completa do azul de metileno em 50 minutos utilizando a condição C4 $\left(55^{\circ} \mathrm{C}, 33 \mathrm{~W}\right)$ utilizando o resíduo agroindustrial, que foi a melhor condição experimental do presente estudo. Estudos realizados por Moraes et al. (2017) utilizando POA acoplado com CA revelaram que o mecanismo de degradação de algumas moléculas é predominantemente na fase homogênea, mas uma parte ocorre na superfície do carvão ativado, mostrando que este 
material adsorvente contribui significativamente para o aumento da taxa de remoção do fenol. Repulsão da molécula com os sítios presentes na superfície do Carvão ativado.

Os resultados obtidos com o carvão ativado $\mathrm{AC}$, mostrou-se menor eficiência na remoção do $\mathrm{MB}$, onde houve uma eliminação mais lenta, quando comparados com os resíduos da casca de banana, porém isto também irá depender condição experimental. Os melhores resultados com a acoplagem POA UV/Shell foi no experimento $\mathrm{C} 2\left(55{ }^{\circ} \mathrm{C}, 15 \mathrm{~W}\right)$ onde a temperatura foi o parâmetro decisivo no processo de eliminação do MB. As menores taxas de eliminação foram encontrados nas condições $\mathrm{C} 1\left(15^{\circ} \mathrm{C}, 15 \mathrm{~W}\right)$ e $\mathrm{C} 3\left(15^{\circ} \mathrm{C}, 33 \mathrm{~W}\right)$, ambos utilizando uma temperatura de $15^{\circ} \mathrm{C}$. A diferença entre a eficiência do POA utilizando diferentes materiais é justificada pelas propriedades do CA ou do material adsorvente utilizado (área superficial e concentrações de groupos ácidos ou básicos presentes da surface do CA) que desempenharam um papel significativo no grau de melhoria (Medellin-Castillo et al., 2013), e neste estudo podendo haver repulsão da molécula poluente com os sítios presentes na superfície do Carvão ativado, resultados que serão confirmados posteriormente com as anpalises IRTF e MEV.

Alguns autores também obtiveram resultados semelhantes utilizando POA/AC onde uma quantidade maior de funções tanto ácidas (Valdés e Zaror, 2006) como básicas (Sanchez-polo et al., 2005) podem aumentar a interação do CA com o agente oxidante aumentando desta forma a geração de radicais hidroxilas •OH (Flouret at al., 2018; Oliveira et al., 2011;). Outros estudos que acoplaram carvão ativado em Processos Oxidativos Avançados também concluiram que AC apresentam uma opção atraente para aumentar a eficiência de eliminação, quando comparados com processos separados, como foi obtido no presente estudo (Moraes et al. (2017); Dabek et al., 2012a; 2012b).

A Tabela 5 apresenta as respostas obtidas no tratamento por POA UV/AC e UV/Cascas, taxa de eliminação de DEP $\left(\mathrm{Y}_{1}\right)$, e o tempo da foto-oxidação total para $100 \%$ do micropoluente $\left(\mathrm{Y}_{2}\right)$, e a Figura 3 a análise de superfície de resposta para os experimentos com azul de metileno (MB) UV/AC.

Tabela 5. Respostas da taxa de eliminação do azul de metileno (MB) (\%) pelo POA.

\begin{tabular}{ccccc}
\hline Ensaios & x1 (lâmpada) & $\begin{array}{c}\text { X2 } \\
\text { (tempertaura) }\end{array}$ & $\begin{array}{c}\text { Taxa de } \\
\text { eliminação } \\
(\%)\end{array}$ & $\begin{array}{c}\text { Taxa de } \\
\text { eliminação } \\
\text { UV/ AC }\end{array}$ \\
\hline 1 & 11 & 15 & 50,18 & UV/ Cascas \\
\hline 2 & 11 & 55 & 81,79 & 65,34 \\
3 & 33 & 15 & 77,58 & 68,38 \\
4 & 33 & 55 & 60,72 & 43,47 \\
5 & 22 & 35 & 55,93 & 90,42 \\
6 & 22 & 35 & 50,35 & 75,09 \\
7 & 22 & 35 & 52,15 & 78,28 \\
\hline
\end{tabular}

Fonte: Autores. 
Figura 3. Superfície de resposta para Taxa de eliminação de MB (\%) por POA em função da temperatura and potência da lâmpada.

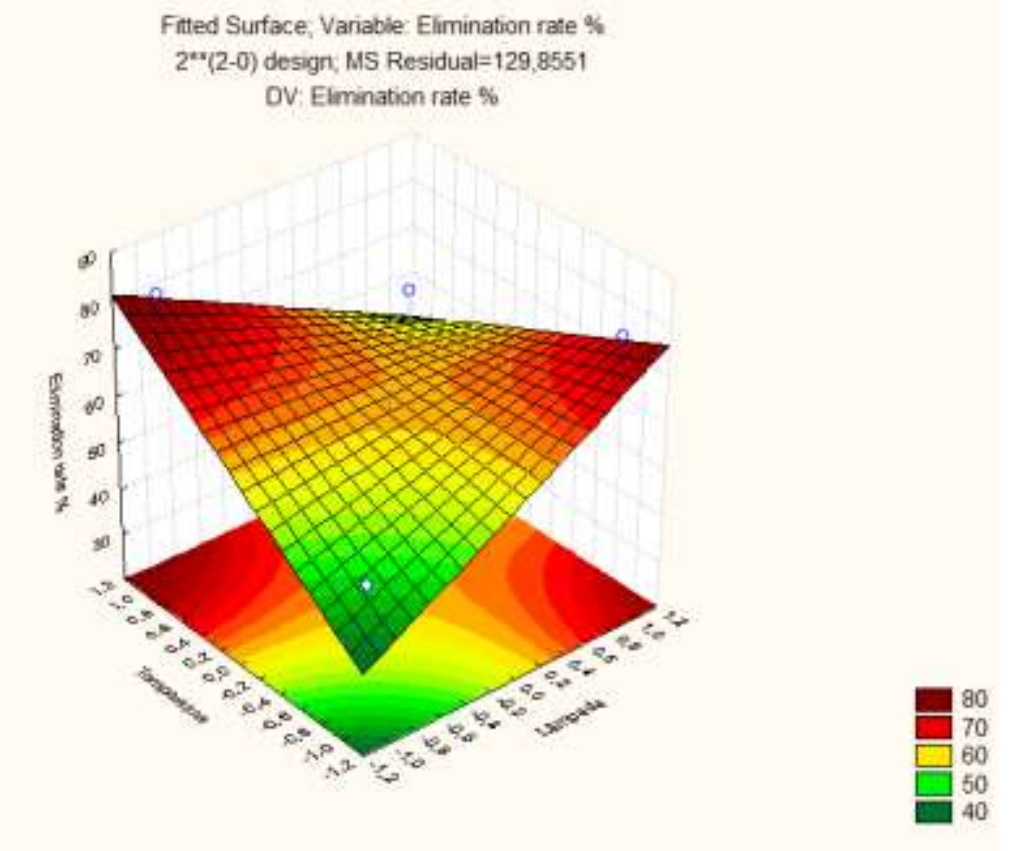

Fonte: Autores.

As superfícies de resposta (Figura 3) confirmam os dados obtidos experimentalmente, onde os valores maiores para a taxa de eliminação de MB por POA ocorreram para os maiores valores de temperatura do processo, com taxa de eliminação de 90,42\% para a condição C4. Observa-se também que o efeito das variáveis observado na Tabela 4 é positivo, para esta variável, ou seja, diretamente proporcional, quando há aumento da temperatura, tem-se aumento da taxa de eliminação do poluente MB no processo. Tanto a temperature como a intensidade da lâmpada juntas tem efeitos positivos na taxa de eliminação do $\mathrm{MB}$, o que foi observado nas condições medianas $\left(35^{\circ} \mathrm{C}\right.$ e $\left.22 \mathrm{~W}\right)$ onde houve uma taxa de eliminação elevada (superior a $70 \%)$ e diferem estatisticamente $(\mathrm{p}<0.05)$.

Tabela 6. Estimativa de efeitos de variáveis em função da taxa de eliminação.

\begin{tabular}{lll}
\hline Variáveis & \multicolumn{2}{l}{ Taxa de eliminação de MB (\%) } \\
\cline { 2 - 3 } & Efeito & $\mathrm{p}$ \\
\hline Temperatura & 3.1650 & 0.563 \\
Potência (W) & 7.3750 & 0.799 \\
Interação & 61.2429 & 0.0007 \\
\hline
\end{tabular}

Significância p>0.05. Fonte: Autores. 


\subsection{Caracterização dos bioadsorventes antes e após os tratamentos}

\subsubsection{Análise IRTF e MEV}

Os resultados da análise de IRTF estão apresentados na Figura 4. O método IRTF permitiu uma estimativa qualitativa dos grupos funcionais, com maior absorção é entre 1500 e 1800 cm-1, que representam os grupos quinona, lactona e carbonila. Grandes quantidades de grupos oxigenados entre $1710 \mathrm{~cm}-1$ e $1750 \mathrm{~cm}-1$, ligação C=O, grupos ácido carboxílico, anidro e lactonas (Oliveira et al., 2011b). Entre 1100 e 1250 cm-1, a ligação C-O (1125: ácido anidro, 1175: fenol) e entre 1500 e 1800 cm-1 (carbonila e quinona). Na Figura 2, as letras (A), (B) e (C) representam, respectivamente, o comprimento de onda de adsorção para: (A) anidridos de ácido fenol; (B) ácido carboxílico, anidrido e lactona; e (C) grupos carbonila e de quinona. Deste modo, o IRTF revela a predominância de grupos carbonila e de quinona, grupos importantes para adsorção de DEP pelo CA. Absorção situada em 1749 cm-1 é característica de estiramento vibracional de $\mathrm{C}=\mathrm{O}$ de carbonila, que é vibração de deformação axial da ligação C-O, já a que aparece próximo a $1168 \mathrm{~cm}-1$, sugere a presença tanto de ácidos carboxílicos como de ésteres ou $\delta$-lactonas ligados a grupos aromáticos (Guilarduci et al., 2006). As absorções em 1600 e $1480 \mathrm{~cm}-1$ presentes em ambos os CAs são referentes ao estiramento $\mathrm{C}=\mathrm{C}$ de aromáticos (Ahmad et al., 2007).

Figura 4. Espectro na região do infravermelho (FTIR) dos materiais utilizados.

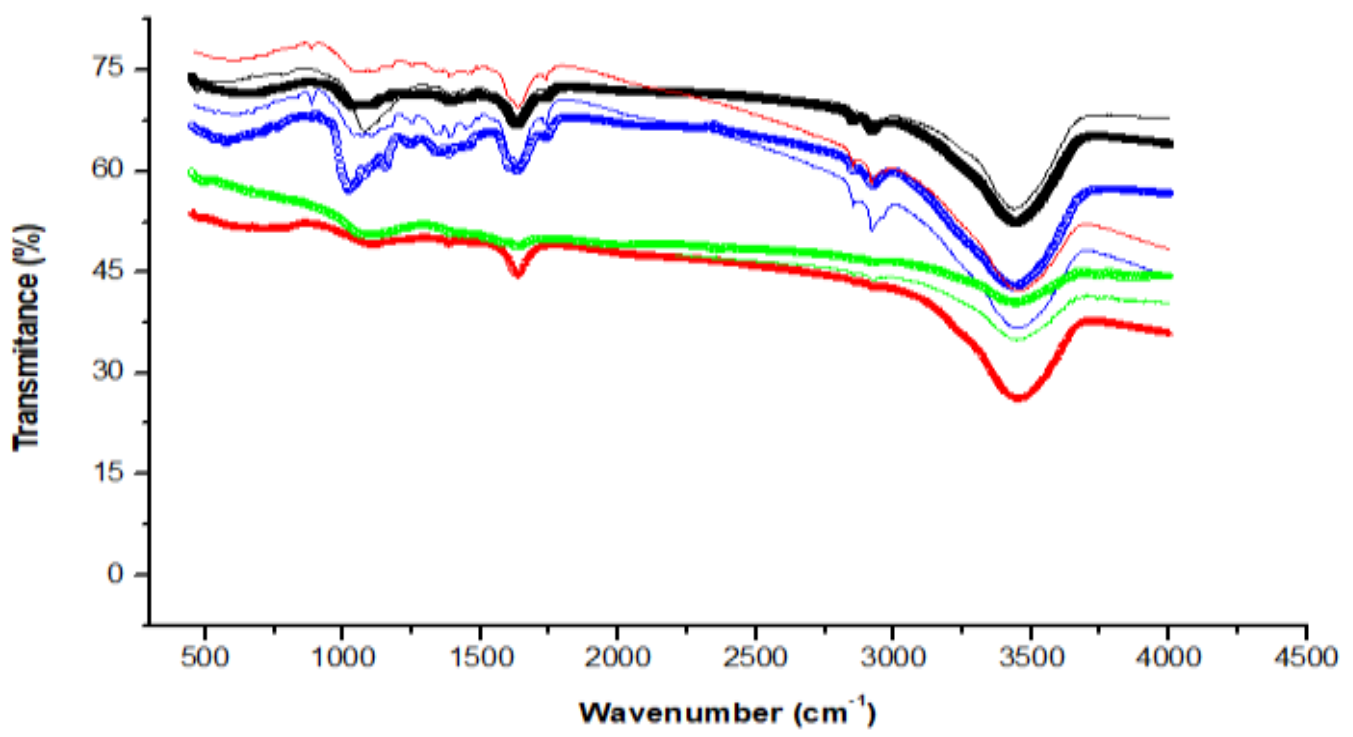

Legenda: • (Casca de mandioca após POA/UC) ; • (Casca de mandioca após adsorção); • (Casca de mandioca seca); -- (Casca de banana após POA/UC); -- (Casca de banana após adsorção); -- (Casca de banana seca); • (CA original); - (CA após POA/UC).

Fonte: Autores.

A estrutura de CA, pré e pós processo UV/ foram determinadas (Figura 5). Os resultados revelam que as imagens de MEV de CA pré uso, e pós utilização é ainda suave. Algumas partículas de Carbono do CA antes do uso nos tratamentos são difíceis de encontrar nas imagens de MEV de CA utilizado. No entanto, a estrutura de poros de CA não foi danificada, deste modo, obviamente, e as propriedades texturais foram mantidas. Então, pode-se confirmar que de acordo com Zhang et al. (2014), que em um processo de reutilização de CA, por longo tempo não altera, significativamente, suas propriedades de texturas. 
Research, Society and Development, v. 10, n. 5, e27310512830, 2021

(CC BY 4.0) | ISSN 2525-3409 | DOI: http://dx.doi.org/10.33448/rsd-v10i5.12830

Figura 5. Imagens de MEV de bioadsorventes pré cinéticas (a, b, banana), (c, d, mandioca), pós cinéticas de acoplagem C4 (e, f, banana; g, h carvão ativado comercial).

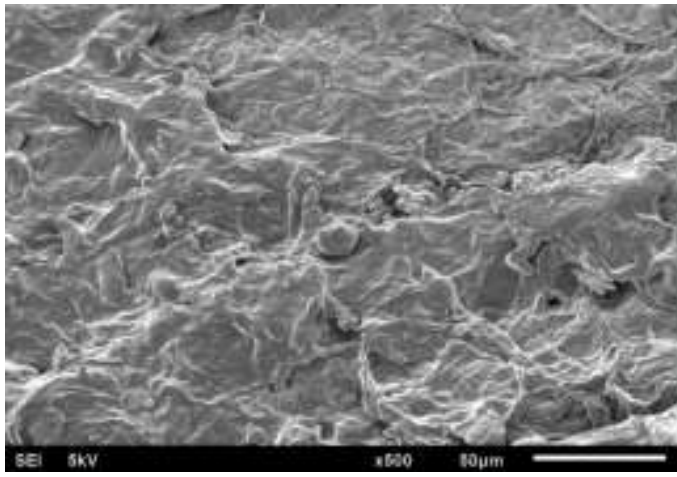

(a)

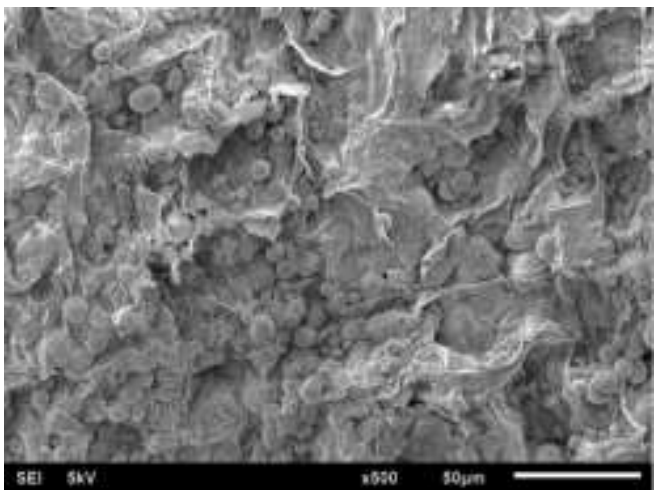

(c)

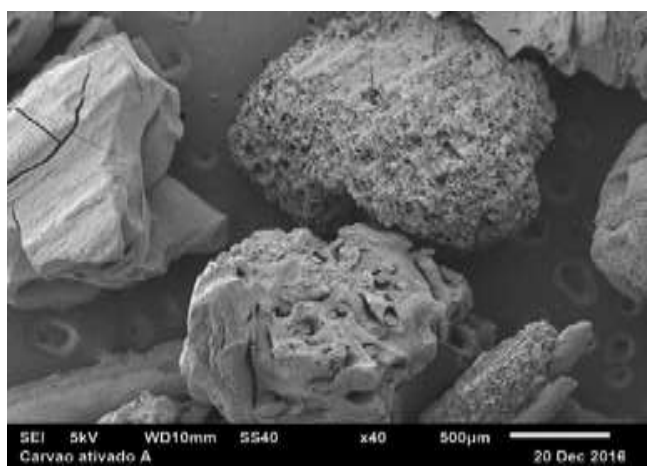

(e)

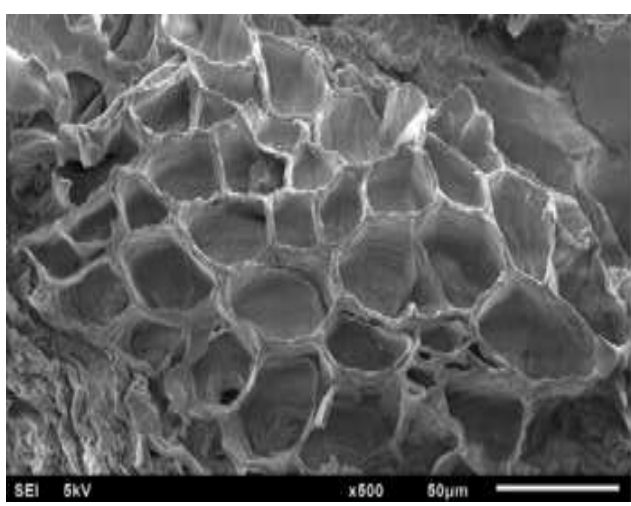

$(\mathrm{g})$

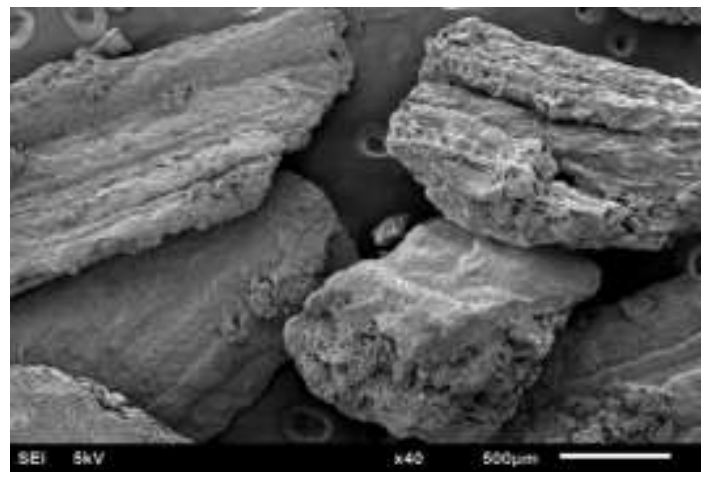

(b)

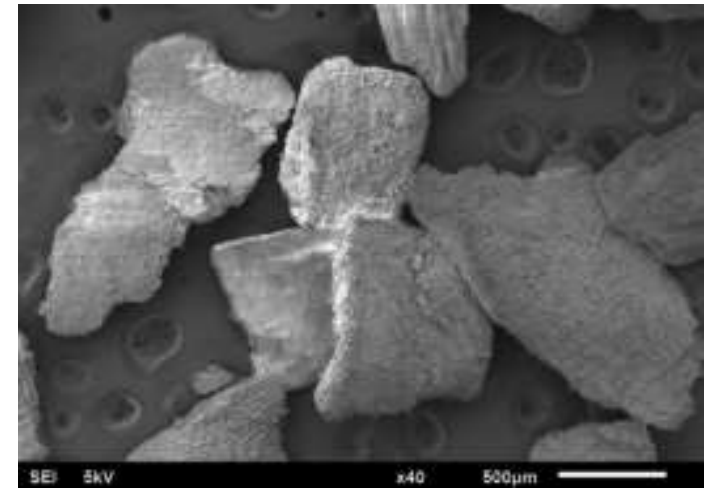

(d)

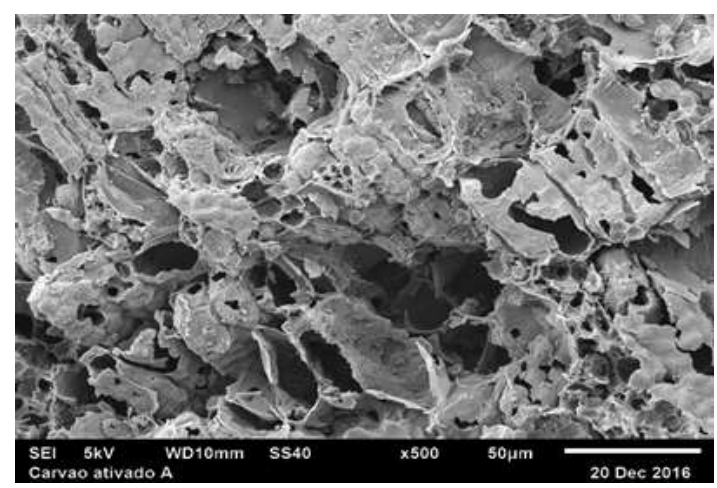

(f)

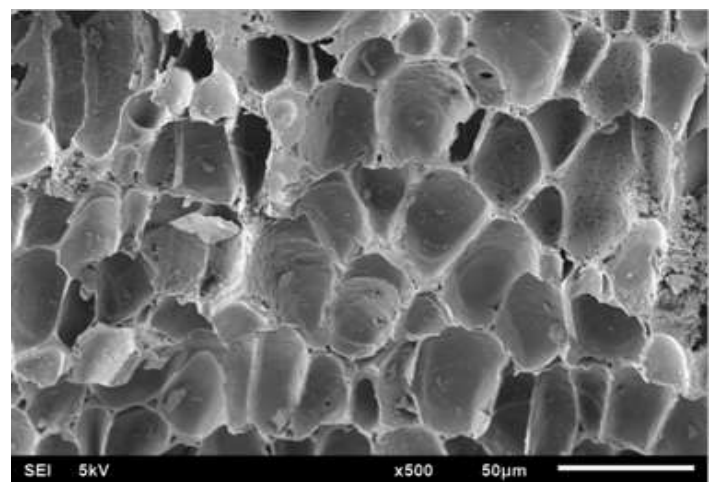

(h)

Fonte: Autores. 


\section{Conclusão}

Este estudo utilizando resíduos de alimentos, na maioria das vezes não reaproveitados, demostra o forte potencial de utilização destes resíduos para outros fins, como demostrado neste estudo em forma de bioadsorventes. Entre os Processos utilizados, processo de adsorção e Processos Oxidativos Avançados utilizando UV, demostraram processos promissores para a redução do poluente modelo, o azul de metileno. Os melhores resultados nos processos de adsorção foram aqueles utilizando o bioadsorvente casca de banana, com uma massa de 0,5 g e uma temperatura de $50{ }^{\circ} \mathrm{C}$. Para os POAs utilizados, a melhor cinética de degradação foi na condição $\mathrm{C} 4\left(55^{\circ} \mathrm{C}, 33 \mathrm{~W}\right)$ utilizando o resíduo casca de banana, contribuindo para a sustentabilidade agroindustrial e para a melhoria dos parâmetros de qualidade da água.

\section{Agradecimentos}

À Capes (Fundação de Amparo à Pesquisa do Estado de Goiás pela bolsa de estudos de doutorado Processo N. 88887.475331/2020-00; ao CNPQ pela bolsa de Iniciação Científica IC (Processo Universal N. 405496/2018-4) e à empresa FBC (Fábrica Brasileira de Catalisadores), que forneceu o carvão ativado.

\section{Referências}

Aguiar, L. (2006). Avaliação do processo de concentração osmótica para obtenção de banana-passa. $2006.103 f$ (Doctoral dissertation, Dissertação (Mestrado em Tecnologia de Alimentos) -Faculdade de Engenharia de Alimentos, Universidade Estadual de Campinas, Campinas).

Almeida, M. C., de Oliveira, T. F., \& de Sa, F. P. (2020). The elimination of cancerous pollutants by an advanced oxidation processes and adsorption in monosolute solutions mixtures in water. Desalination and water treatment, 191, 292-299.

Ahmad, A. L., Loh, M. M., \& Aziz, J. A. (2007). Preparation and characterization of activated carbon from oil palm wood and its evaluation on methylene blue adsorption. Dyes and pigments, 75(2), 263-272.

Ali, H. (2010). Biodegradation of synthetic dyes - a review. Water, Air, \& Soil Pollution, 213(1), 251-273.

Alver, E., Metin, A. Ü., \& Brouers, F. (2020). Methylene blue adsorption on magnetic alginate/rice husk bio-composite. International journal of biological macromolecules, 154, 104-113.

Asfaram, A., Ghaedi, M., Dashtian, K., \& Ghezelbash, G. R. (2018). Preparation and characterization of Mn0. 4Zn0. 6Fe2O4 nanoparticles supported on dead cells of Yarrowia lipolytica as a novel and efficient adsorbent/biosorbent composite for the removal of azo food dyes: central composite design optimization study. ACS Sustainable Chemistry \& Engineering, 6(4), 4549-4563.

Asfour, H. M., Fadali, O. A., Nassar, M. M., \& El-Geundi, M. S. (1985). Equilibrium studies on adsorption of basic dyes on hardwood. Journal of Chemical Technology and Biotechnology. Chemical Technology, 35(1), 21-27.

Belaid, K. D., Kacha, S., Kameche, M., \& Derriche, Z. (2013). Adsorption kinetics of some textile dyes onto granular activated carbon. Journal of Environmental Chemical Engineering, 1(3), 496-503.

Boehm, H. P. (2002). Surface oxides on carbon and their analysis: a critical assessment. Carbon, 40(2), 145-149.

Coha, M., Farinelli, G., Tiraferri, A., Minella, M., \& Vione, D. (2021). Advanced oxidation processes in the removal of organic substances from produced water: Potential, configurations, and research needs. Chemical Engineering Journal, 128668.

Dąbek, L., Ozimina, E., \& Picheta-Oleś, A. (2012). Applying the combined processes of sorption and oxidation to remove organic compounds from an aqueous environment using the example of p-chlorophenol. Ecological Chemistry and Engineering. A, 19(3), 275-286.a

Dabek, L., Ozimina, E., \& Picheta-Oles, A. (2012). Dye removal efficiency of virgin activated carbon and activated carbon regenerated with Fenton's reagent. Environment Protection Engineering, 38(1), 5-13.b

Doğan, M., \& Alkan, M. (2003). Adsorption kinetics of methyl violet onto perlite. Chemosphere, 50(4), 517-528.

Ezzatahmadi, N., Ayoko, G. A., Millar, G. J., Speight, R., Yan, C., Li, J., ... \& Xi, Y. (2017). Clay-supported nanoscale zero-valent iron composite materials for the remediation of contaminated aqueous solutions: a review. Chemical engineering journal, 312, 336-350.

Fadillah, G., Saleh, T. A., Wahyuningsih, S., Putri, E. N. K., \& Febrianastuti, S. (2019). Electrochemical removal of methylene blue using alginate-modified graphene adsorbents. Chemical Engineering Journal, 378, 122140.

Flouret, A., de Almeida, M. C., de Oliveira, T. F., \& de Sá, F. P. (2018). Advanced treatment of phenol by H2O2/UV/activated carbon coupling: Influence of homogeneous and heterogeneous phase. The Canadian Journal of Chemical Engineering, 96(9), 1979-1985. 
Habib, I. Y., Burhan, J., Jaladi, F., Lim, C. M., Usman, A., Kumara, N. T. R. N., ... \& Mahadi, A. H. (2020). Effect of Cr doping in CeO2 nanostructures on photocatalysis and $\mathrm{H} 2 \mathrm{O} 2$ assisted methylene blue dye degradation. Catalysis Today.

Iheukwumere, F. C., Ndubuisi, E. C., Mazi, E. A., \& Onyekwere, M. U. (2008). Performance, nutrient utilization and organ characteristics of broilers fed cassava leaf meal (Manihot esculenta Crantz). Pakistan Journal of Nutrition, 7(1), 13-16.

Islam, M. A., Ali, I., Karim, S. A., Firoz, M. S. H., Chowdhury, A. N., Morton, D. W., \& Angove, M. J. (2019). Removal of dye from polluted water using novel nano manganese oxide-based materials. Journal of Water Process Engineering, 32, 100911.

Kousha, M., Daneshvar, E., Dopeikar, H., Taghavi, D., \& Bhatnagar, A. (2012). Box-Behnken design optimization of Acid Black 1 dye biosorption by different brown macroalgae. Chemical Engineering Journal, 179, 158-168.

Li, Z., Hanafy, H., Zhang, L., Sellaoui, L., Netto, M. S., Oliveira, M. L., ... \& Li, Q. (2020). Adsorption of congo red and methylene blue dyes on an ashitaba waste and a walnut shell-based activated carbon from aqueous solutions: Experiments, characterization and physical interpretations. Chemical Engineering Journal, 388, 124263 .

Li, Z., Sellaoui, L., Dotto, G. L., Lamine, A. B., Bonilla-Petriciolet, A., Hanafy, H., ... \& Erto, A. (2019). Interpretation of the adsorption mechanism of Reactive Black 5 and Ponceau 4R dyes on chitosan/polyamide nanofibers via advanced statistical physics model. Journal of Molecular Liquids, 285, 165-170.

Moraes, M. F., de Oliveira, T. F., Cuellar, J., \& Castiglioni, G. L. (2017). Phenol degradation using adsorption methods, advanced oxidative process (H2O2/UV) and H2O2/UV/activated carbon coupling: influence of homogeneous and heterogeneous phase. Desalination and Water Treatment, 100, 38-45.

Medellin-Castillo, N. A., Ocampo-Pérez, R., Leyva-Ramos, R., Sanchez-Polo, M., Rivera-Utrilla, J., \& Méndez-Díaz, J. D. (2013). Removal of diethyl phthalate from water solution by adsorption, photo-oxidation, ozonation and advanced oxidation process (UV/H2O2, O3/H2O2 and O3/activated carbon). Science of the total environment, 442, 26-35.

Mittal, A., Mittal, J., \& Kurup, L. (2006). Adsorption isotherms, kinetics and column operations for the removal of hazardous dye, Tartrazine from aqueous solutions using waste materials_-Bottom Ash and De-Oiled Soya, as adsorbents. Journal of hazardous materials, 136(3), 567-578.

Moraes, J. T., Salamanca-Neto, C. A. R., Švorc, L., \& Sartori, E. R. (2017). Advanced sensing performance towards simultaneous determination of quaternary mixture of antihypertensives using boron-doped diamond electrode. Microchemical Journal, 134, 173-180.

de Oliveira, T. F., Chedeville, O., Cagnon, B., \& Fauduet, H. (2011). Degradation kinetics of DEP in water by ozone/activated carbon process: Influence of pH. Desalination, 269(1-3), 271-275.

Pang, X., Sellaoui, L., Franco, D., Dotto, G. L., Georgin, J., Bajahzar, A., ... \& Li, Z. (2019). Adsorption of crystal violet on biomasses from pecan nutshell, para chestnut husk, araucaria bark and palm cactus: experimental study and theoretical modeling via monolayer and double layer statistical physics models. Chemical Engineering Journal, 378, 122101.

Parvin, S., Rahman, M. W., Saha, I., Alam, M. J., \& Khan, M. M. R. (2019). Coconut tree bark as a potential low-cost adsorbent for the removal of methylene blue from wastewater. DESALIN WATER TREAT, 146, 385-392.

Ponnusami, V., Vikram, S., \& Srivastava, S. N. (2008). Guava (Psidium guajava) leaf powder: novel adsorbent for removal of methylene blue from aqueous solutions. Journal of hazardous materials, 152(1), 276-286.

Reddy, P. M. K., Verma, P., \& Subrahmanyam, C. (2016). Bio-waste derived adsorbent material for methylene blue adsorption. Journal of the Taiwan Institute of Chemical Engineers, 58, 500-508.

Rivera-Utrilla, J., \& Sánchez-Polo, M. (2002). Ozonation of 1, 3, 6-naphthalenetrisulphonic acid catalysed by activated carbon in aqueous phase. Applied Catalysis B: Environmental, 39(4), 319-329.

Rivera-Utrilla, J., Méndez-Díaz, J., Sánchez-Polo, M., Ferro-García, M. A., \& Bautista-Toledo, I. (2006). Removal of the surfactant sodium dodecylbenzenesulphonate from water by simultaneous use of ozone and powdered activated carbon: Comparison with systems based on O3 and $\mathrm{O} 3 / \mathrm{H} 2 \mathrm{O} 2$. Water research, $40(8), 1717-1725$.

Sousa, D. N. R., Insa, S., Mozeto, A. A., Petrovic, M., Chaves, T. F., \& Fadini, P. S. (2018). Equilibrium and kinetic studies of the adsorption of antibiotics from aqueous solutions onto powdered zeolites. Chemosphere, 205, 137-146.

Sánchez-Polo, M., von Gunten, U., \& Rivera-Utrilla, J. (2005). Efficiency of activated carbon to transform ozone into OH radicals: influence of operational parameters. Water research, 39(14), 3189-3198.

Santhosh, C., Velmurugan, V., Jacob, G., Jeong, S. K., Grace, A. N., \& Bhatnagar, A. (2016). Role of nanomaterials in water treatment applications: a review. Chemical Engineering Journal, 306, 1116-1137.

Shooto, N. D., Thabede, P. M., Bhila, B., Moloto, H., \& Naidoo, E. B. (2020). Lead ions and methylene blue dye removal from aqueous solution by mucuna beans (velvet beans) adsorbents. Journal of Environmental Chemical Engineering, 8(2), 103557.

Somsesta, N., Sricharoenchaikul, V., \& Aht-Ong, D. (2020). Adsorption removal of methylene blue onto activated carbon/cellulose biocomposite films: equilibrium and kinetic studies. Materials Chemistry and Physics, 240, 122221.

Stoeckli, F., López-Ramón, M. V., Hugi-Cleary, D., \& Guillot, A. (2001). Micropore sizes in activated carbons determined from the Dubinin-Radushkevich equation. Carbon, 39(7), 1115-1116.

Uddin, M. T., Islam, M. A., Mahmud, S., \& Rukanuzzaman, M. (2009). Adsorptive removal of methylene blue by tea waste. Journal of Hazardous Materials, 164(1), 53-60. 
Research, Society and Development, v. 10, n. 5, e27310512830, 2021

(CC BY 4.0) | ISSN 2525-3409 | DOI: http://dx.doi.org/10.33448/rsd-v10i5.12830

Valdés, H., \& Zaror, C. A. (2006). Heterogeneous and homogeneous catalytic ozonation of benzothiazole promoted by activated carbon: kinetic approach. Chemosphere, 65(7), 1131-1136.

Vieira Filho, J. E. R., \& Fishlow, A. (2017). Agricultura e indústria no Brasil: inovação e competitividade.

Yagub, M. T., Sen, T. K., Afroze, S., \& Ang, H. M. (2014). Dye and its removal from aqueous solution by adsorption: a review. Advances in colloid and interface science, 209, 172-184.

Zare, E. N., Lakouraj, M. M., \& Ramezani, A. (2016). Efficient sorption of Pb (II) from an aqueous solution using a poly (aniline-co-3-aminobenzoic acid)based magnetic core-shell nanocomposite. New Journal of Chemistry, 40(3), 2521-2529.

Zhang, T., Walawender, W. P., Fan, L. T., Fan, M., Daugaard, D., \& Brown, R. C. (2004). Preparation of activated carbon from forest and agricultural residues through CO2 activation. Chemical Engineering Journal, 105(1-2), 53-59.

Zhang, W., Zhou, C., Zhou, W., Lei, A., Zhang, Q., Wan, Q., \& Zou, B. (2011). Fast and considerable adsorption of methylene blue dye onto graphene oxide. Bulletin of environmental contamination and toxicology, 87(1), 86-90.

Zhou, Y., Lu, J., Zhou, Y., \& Liu, Y. (2019). Recent advances for dyes removal using novel adsorbents: a review. Environmental pollution, $252,352-365$. 